\title{
Avoided level crossing and exceptional points
}

\author{
W D Heiss $\$$ and A L Sanninoł \\ † W K Kellogg Radiation Laboratory, California Institute of Technology, Pasadena, CA \\ 91125, USA \\ $\ddagger$ Department of Physics and Centre for Nonlinear Studies, University of the Witwatersrand, \\ Johannesburg, 2050, South Africa
}

Received 13 June 1989, in final form 19 September 1989

\begin{abstract}
The connection between level repulsions and the singularities associated with the analytically continued energy levels is investigated. We also conjecture that there are necessarily specific consequences for the state vectors when the statistical analysis of the energy spectrum indicates quantum chaos. A procedure which allows a qualitative assessment of the positions of the exceptional points is suggested. The importance of their distribution for quantum chaos is discussed within this context.
\end{abstract}

\section{Introduction}

Avoided level crossings have always played an important role in quantum mechanics such as nuclear physics [1] but also in quantum electrodynamics [2], as well as in quantum chromodynamics [3]. More recently, the subject of quantum chaos has attracted particular interest; it is directly linked to avoided level crossings. This paper is motivated by the consideration that if specific Hamiltonians in quantum mechanics are distinct from others by manifesting patterns that are denoted as quantum chaos, such properties must reside with the operator properties of the Hamiltonian. If successful, such an approach should eventually even give rise to a satisfactory definition of quantum chaos.

Since quantum chaos is, in contrast to its classical counterpart, at best vaguely defined [4], a short discussion seems to be appropriate. There are the two traditional approaches: the statistical approach using Gaussian orthogonal ensembles of Hamiltonians (GOE) to obtain statements about the statistical properties of the spectrum [5]. There is no physical input $a$ priori and the major motivation lies in the successful description of nuclear spectra at high level density. On the other side is the semiclassical approach which relies on quantisation of classically chaotic systems [6]. Clearly, such systems must be sufficiently small to allow analytic and/or numerical treatment of the analogous classical case. With regard to the spectral properties a connection has been established between the two approaches [7]. A great deal of insight and applications have been obtained [8] from these efforts, but a number of questions are still outstanding.

§ On leave of absence from the Department of Physics, University of the Witwatersrand, Johannesburg, South Africa. 
One of these questions concerns the specific property of the quantum mechanical operator that gives rise to quantum chaos. This, however, throws us back to the question: what is quantum chaos? A tentative answer may be provided by a mathematical model [9] for a scattering situation. For a physical bound-state problem an answer cannot in all cases rely on the classical analogy, for a number of reasons. For instance, due to its complexity, a many body system is not amenable to detailed analysis by Newtonian mechanics. Also, typical quantum effects like the existence of a good mean field and collective motion are manifestations of order in a quantum system whose classical counterpart is chaotic. On the other hand, the statistical properties of only the spectrum do not seem to provide a satisfactory criterion either. We believe that a connection should be sought between the spectral properties and a specific behaviour of the corresponding state vectors. Since an abstract orthogonal basis does not distinguish between a regular and a chaotic Hamiltonian, one has to refer to a particular representation. In the semiclassical approach, reference is naturally made to position representation, i.e. the Schrödinger wavefunction. In a general quantum system, like a many body system, there seems to be no choice but to rely on matrix elements of physical operators such as the momentum operator. In this way one can hope in principle to reduce the criteria for quantum chaos to the properties of the quantum mechanical Hamiltonian. We do not offer criteria in this paper, but rather address ourselves to the mathematical properties of Hamiltonians in terms of which such criteria could be formulated. Intuitively, we would expect 'erratic' behaviour of matrix elements $\langle m|p| n\rangle$ as a function of $n$ and $m$ when the state vectors refer to a chaotic Hamiltonian; we would expect, at least locally, high sensitivity of levels and matrix elements under perturbation.

Level repulsion is inextricably part of a quantum chaotic spectrum. In order to study level repulsion we need at least one parameter to visualise the repelling levels. A Hamiltonian of the form $H_{0}+\lambda H_{1}$ is the starting point of many investigations in all branches of physics. It gives rise to the spectrum $E_{k}(\lambda)$. We may specifically assume that the two Hamiltonians $H_{0}$ and $H_{1}$ are regular when considered individually. In this case we can interpret the problem as a transition from order to order prevailing for $\lambda=0$ and $\lambda \rightarrow \infty$, respectively. If the two operators are incompatible, the mixed problem is characterised by many level repulsions. We may virtually exclude any level crossing by assuming that the full Hamiltonian is irreducible with respect to internal symmetries.

Each level repulsion is associated with branch-point singularities of the levels when they are continued into the complex $\hat{\lambda}$ plane. The singularities are called exceptional points [10], they are specific to a particular Hamiltonian. Whether or not the spectrum satisfies the criteria in accordance with GOE must depend on the distribution of the exceptional points. In this paper an attempt is made to predict the distribution of the exceptional points only from the knowledge of $H_{0}$ and $H_{1}$ as long as they are represented by finite-dimensional matrices. This is a preliminary attempt in that the underlying idea is presented and no statistical treatment is pursued. Also, the more interesting question about the corresponding behaviour of the state vectors is dealt with only in a cursory manner.

In section 2 we explain the concept of exceptional points in an elementary way using two-dimensional matrices. The non-trivial extension to higher dimensions is discussed in section 3 . In section 4 the actual procedure for obtaining knowledge about the distribution of the exceptional points is presented. A discussion concludes the paper. 


\section{Analytic treatment for a two-dimensional matrix}

We use a $2 \times 2$ matrix to illustrate in an elementary way the concept and the parametric dependence of the exceptional points. The problem investigated is of the form $H_{0}+\hat{\lambda} H_{1}$.

We assume $H_{0}$ to be diagonal with eigenvalues $\varepsilon_{1}$ and $\varepsilon_{2}$ with $\varepsilon_{1}<\varepsilon_{2}$. The eigenvalues of $H_{1}$ are denoted by $\omega_{1}$ and $\omega_{2}, \omega_{1}<\omega_{2}$. If the two operators commute, i.e. if $H_{1}$ is also diagonal, the eigenvalues of the combined problem are

$$
E_{k}^{0}(\lambda)=\varepsilon_{k}+\lambda \omega_{k} \quad k=1,2 .
$$

The two lines $E_{k}^{0}(\lambda)$ intersect at $\lambda=\lambda_{0}=-\left(\varepsilon_{1}-\varepsilon_{2}\right) /\left(\omega_{1}-\omega_{2}\right)$, where the two levels are degenerate. This degeneracy is lifted if the two levels in $H_{1}$ are coupled. This can be accomplished by a rotation of $H_{1}$. In this way we preserve the spectrum of $H_{1}$. Denoting the diagonal representation of $H_{1}$ by $D$, we use $H_{1}=U D U^{-1}$, which rotates $D$ to the representation in which $H_{0}$ is diagonal. The rotation matrix is of the form

$$
U=\left(\begin{array}{cc}
\cos \varphi & -\sin \varphi \\
\sin \varphi & \cos \varphi
\end{array}\right)
$$

The eigenvalues of the problem $H_{0}+\lambda H_{1}$ are

$$
E_{1}(\lambda)=\frac{\varepsilon_{1}+\varepsilon_{2}+\lambda\left(\omega_{1}+\omega_{2}\right)}{2}-R \quad E_{2}(\lambda)=\frac{\varepsilon_{1}+\varepsilon_{2}+\lambda\left(\omega_{1}+\omega_{2}\right)}{2}+R
$$

where

$$
R=\left[\left(\frac{\varepsilon_{1}-\varepsilon_{2}}{2}\right)^{2}+\left(\frac{\lambda\left(\omega_{1}-\omega_{2}\right)}{2}\right)^{2}+\frac{1}{2} \lambda\left(\varepsilon_{1}-\varepsilon_{2}\right)\left(\omega_{1}-\omega_{2}\right) \cos 2 \varphi\right]^{1 / 2}
$$

Clearly, for $\varphi \neq 0$ there is level repulsion of the two levels. If $\varphi$ is small the level repulsion occurs in the vicinity of $\lambda_{0}$. The level repulsion is associated with the exceptional points which are the branch point singularities of $E_{k}(\lambda)$ when the two functions $E_{k}$ are continued in the variable $\lambda$. The exceptional points occur at the zeros of the square root in (1), which are positioned at the two complex conjugate values

$$
\lambda_{c}=-\frac{\varepsilon_{1}-\varepsilon_{2}}{\omega_{1}-\omega_{2}} \exp ( \pm 2 i \varphi)
$$

Obviously, the two functions $E_{k}(\lambda)$ are the values of one single analytic function on two Riemann sheets. The two sheets are connected at the square root branch point singularities occurring at $\lambda=\lambda_{\mathrm{c}}$. At those points the values of the two functions are equal, i.e. $E_{1}\left(\lambda_{c}\right)=E_{2}\left(\lambda_{c}\right)$. Only for the particular values of the angles $\varphi=0$ or $\varphi=\pi / 2$ do the two Riemann sheets become disconnected as the two branch points coalesce on the real axis; then the two levels become the functions $E_{1}^{0}(\lambda)$ and $E_{2}^{0}(\lambda)$, which are both without branch point and have no analytic connection; this situation corresponds to the actual degeneracy occurring at $\lambda_{0}$. When $\varphi$ is varied between 0 and $\pi / 2$ the branch points move on a circle according to (2). For illustration, we plot in figure 1 the two levels as a function of $\lambda$ for a few values of $\varphi$. The effect of the position of the exceptional points on the position and the type of the level repulsion is clearly seen: the closer the exceptional point to the real axis, the narrower the mutual approach of 

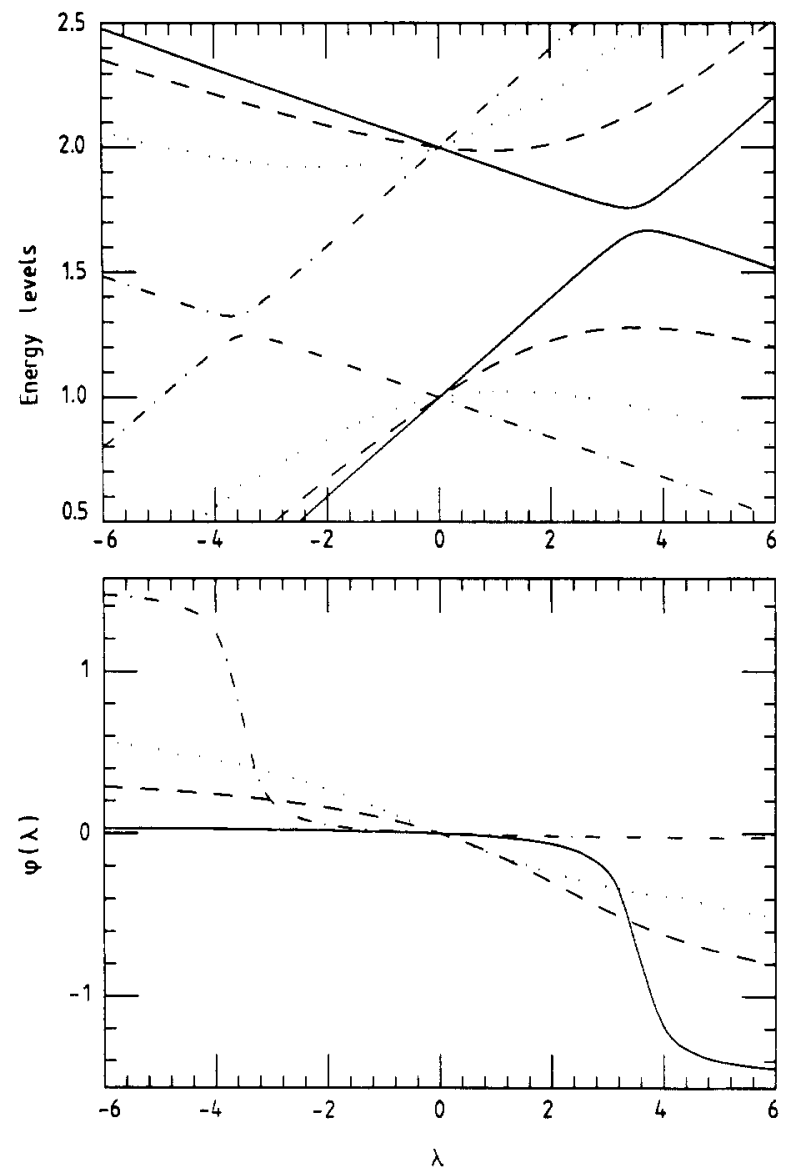

Figure 1. Top: the two levels as a function of $\lambda$ for $\varphi=0.05$ (chain curve), 0.45 (dotted curve), 0.85 (broken curve) and 1.52 (full curve). Bottom: $\varphi(\lambda)$ for the same parameter values.

the two repelling levels. This corresponds to the magnitude of the coupling term in $H_{1}$ which is given by $\frac{1}{2}\left(\omega_{1}-\omega_{2}\right) \sin 2 \varphi$. If the coupling is weak the two levels nearly cross, while they are strongly repelled when the coupling is strong.

Of interest is the behaviour of the eigenvectors which are the column vectors of the orthogonal matrix $U(\lambda)$ that diagonalises $H_{0}+\lambda H_{1}$. Clearly, $U(0)$ is the unit matrix while $U(\infty)$ is identical to $U$ introduced above. We parametrise $U(\lambda)$ by the angle $\varphi(\lambda)$, which is determined by

$$
\tan \varphi(\lambda)=\frac{1}{2} \frac{\lambda\left(\omega_{2}-\omega_{1}\right) \sin 2 \varphi}{E_{2}(\lambda)-\varepsilon_{1}-\lambda\left(\omega_{1} \cos ^{2} \varphi+\omega_{2} \sin ^{2} \varphi\right)} .
$$

This confirms that $\varphi(0)=0$ and $\varphi(\infty)=\varphi$. Furthermore, $\varphi(-\infty)=\varphi(\infty)+\pi / 2$. The decrease of $\varphi(\lambda)$ by $\pi / 2$ occurs essentially where level repulsion occurs. This drop is the more dramatic the closer the exceptional points lie to the real axis. For illustration a few examples are given in figure 1 . The decrease of $\varphi(\lambda)$ by $\pi / 2$ means that the two eigenvectors interchange their position (apart from the sign of one vector), an effect well known in connection with level repulsion. Instead of referring to the eigenvectors, 
we suggest, for the purpose of the discussion in the following sections, to view $U(\lambda)$ as a continuous (two-dimensional) rotation by the angle $\varphi(\lambda)$. This rotation has a strong $\lambda$ dependence in the vicinity of the exceptional points; otherwise it is a slowly varying function of $\lambda$.

\section{Higher dimensions}

Basically, the procedure described in the previous section can be carried over to higher dimensions. We assume $H_{0}$ to be diagonal in an $N$-dimensional space with ordered eigenvalues $\varepsilon_{1}<\varepsilon_{2}<\ldots<\varepsilon_{N}$. To facilitate the discussion, the eigenvalues of $H_{1}$, denoted by $\omega_{k}, k=1, \ldots, N$, are assumed to be all different from each other. If $H_{1}$ were diagonal, the spectrum of $H_{0}+\lambda H_{1}$ would be $E_{k}^{0}(\lambda)=\varepsilon_{k}+\lambda \omega_{k}$. These $N$ lines would generically have $N(N-1) / 2$ different intersection points. Below we refer to these lines as the unperturbed lines. To switch on coupling terms in $H_{1}$ we use, as in the previous section, the form $H_{1}=U D U^{-1}$, where for $N$-dimensional rotations $N(N-1) / 2$ angles are required to determine $U$. They can be defined in many ways. A suitable definition is discussed in the next section.

The switching on of the rotational angles gives rise in general to $N(N-1) / 2$ complex conjugate pairs of exceptional points which are associated with the $N(N-1) / 2$ avoided level crossings that emerge from the actual crossings when all angles are zero. In other words, the $N$ eigenvalues $E_{k}(\lambda)$ are generically values of one analytic function (which is usually not available explicitly) on $N$ Riemann sheets. The $N(N-1)$ exceptional points connect the $N$ Riemann sheets. Their position is determined by the algebraic problem of solving the simultaneous equations

$$
\operatorname{det}\left[\left(E-\varepsilon_{m}\right) \delta_{m, n}-\lambda H_{m, n}^{(1)}\right]=0 \quad \frac{\mathrm{d}}{\mathrm{d} E} \operatorname{det}\left[\left(E-\varepsilon_{m}\right) \delta_{m, n}-\lambda H_{m, n}^{(1)}\right]=0 .
$$

Note that the determinant is an $N$ th-order polynomial in both $E$ and $\lambda$. The first equation is the usual secular equation, while the second equation enforces equality of two roots of the first equation for the same $\lambda$ value. This is in general possible only for complex values of $\lambda$ (and therefore complex $E(\hat{\lambda})$ ). These exceptional points should not be confused with diabolic points [11], where a degeneracy of a self-adjoint operator is considered when at least two real parameters are suitably chosen. The exceptional points are associated with a more rigid mathematical structure of the analytic functions $E_{k}(\lambda)$; the operator $H_{0}+\lambda H_{1}$ is no longer self-adjoint for complex $\lambda$.

It is instructive to visualise the spectrum for very small values of the angles. A general rotation is a product of $N(N-1) / 2$ primitive rotations, where we denote as a primitive rotation a matrix of the form

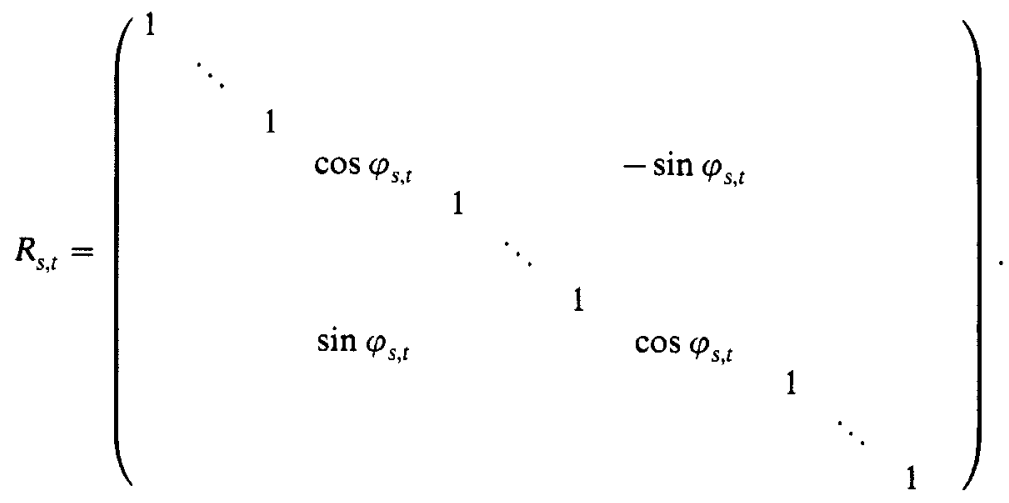


The cos and sin functions occur in the sth and $t$ th row and column. The rotation affects only the $s$ th and $t$ th axes. Consider the special case where $U \equiv R_{s, t}$. From the discussion in the previous section it is obvious that this will cause a level repulsion only between the crossing of the two lines $E_{s}^{0}=\varepsilon_{s}+\hat{\lambda} \omega_{s}$ and $E_{t}^{0}=\varepsilon_{t}+\lambda \omega_{t}$. This observation suggests choosing as primitive rotations precisely the ones which have a one-to-one correspondence to all the crossings of the unperturbed lines. A possible choice is to represent the general rotation by the factors $R_{k, l}$ with $k$ running from 1 to $N-1$ and $l$ running from $k+1$ to $N$. As long as the $N(N-1) / 2$ angles are infinitesimally small, the order of the product of the primitive rotations is of no importance, as this is a secondorder effect. In this way, each of the angles $\varphi_{s, t}$ controls the level repulsion of exactly one crossing of the unperturbed lines labelled by $s$ and $t$. Of course, higher-order effects will give rise to mutual influences.

There is an additional pattern of mutual influence of the positions of exceptional points in dimensions higher than two. Even if only one angle is substantially increased while keeping the others very small, the global pattern of the exceptional points can change dramatically. We illustrate this for the case $N=3$. The spectra displayed in figure 2 are obtained by switching the angle $\varphi_{1,3}$ from approximately 0 to approximately $\pi / 2$ while keeping $\varphi_{1,2}$ and $\varphi_{2,3}$ small. It is clear from figure 2 that the variation of $\varphi_{1,3}$ must affect the anticrossings of the 1-2 lines and the 2-3 lines as well. Trajectories of the exceptional points are obtained from a variation of $\varphi_{1,3}$. In figure 3 the three trajectories in the upper $\lambda$ plane are drawn. The small circles indicate equidistant increase of $\varphi_{1,3}$. The broken and dotted curves refer to the anticrossings of the 2-3 lines and 1-2 lines, respectively. It is clearly discernible how their real parts move towards each other from their starting points at positive real $\lambda$ values before they move further out into the complex plane. This reflects the shrinking distance of the 2-3 and 1-2 anticrossing (compare figures $2(a)$ and $2(b)$ ). It is an obvious geometric consequence caused by the primary level repulsion of the 1-3 lines which is induced by the increase of $\varphi_{1,3}$. The approach of all three exceptional points towards the negative real $\lambda$ axis, when $\varphi_{1,3}$ approaches $\pi / 2$, is reflected in figures $2(c)$ and $2(d)$, in that small level spacings re-emerge. We recall that a symmetric pattern of the trajectories exists in the lower half plane; the dotted curve coalesces at the point $\operatorname{Re} \lambda \approx 2.5$ with its complex conjugate partner; this signals an incidental degeneracy, i.e. for this particular set of parameters the 1 and 2 lines do actually cross.

We finally mention that, in contrast to the two-dimensional case, an increment of an angle $\varphi_{s, t}$ by $\pi$ does not, in general, restore the Hamiltonian $H_{1}$, owing to the noncommutativity of the primitive rotations. Furthermore, when tracing the trajectories while one angle varies continuously from $\varphi_{0}$ to $\varphi_{0}+2 \pi$ the trajectories do not necessarily close as one might naively expect. While of course the physical situation is restored, i.e. the spectrum and wavefunctions (the latter up to a possible sign factor) are the same, a permutation of the positions of the exceptional points may have occurred. Clearly, the geometrical structure of the Riemann surfaces is exactly restored as well.

\section{Generalisation}

For a given $U$ the actual values of the angles $\varphi_{s, t}$ depend on the order of the primitive rotations. Here we define an order that is suitable for an algorithm which retrieves the angles from a given $U$. We fix the order by

$$
U=B_{1} B_{2} \cdots B_{N-1} \quad B_{m}=R_{m, m+1} R_{m, m+2} \cdots R_{m, N} .
$$



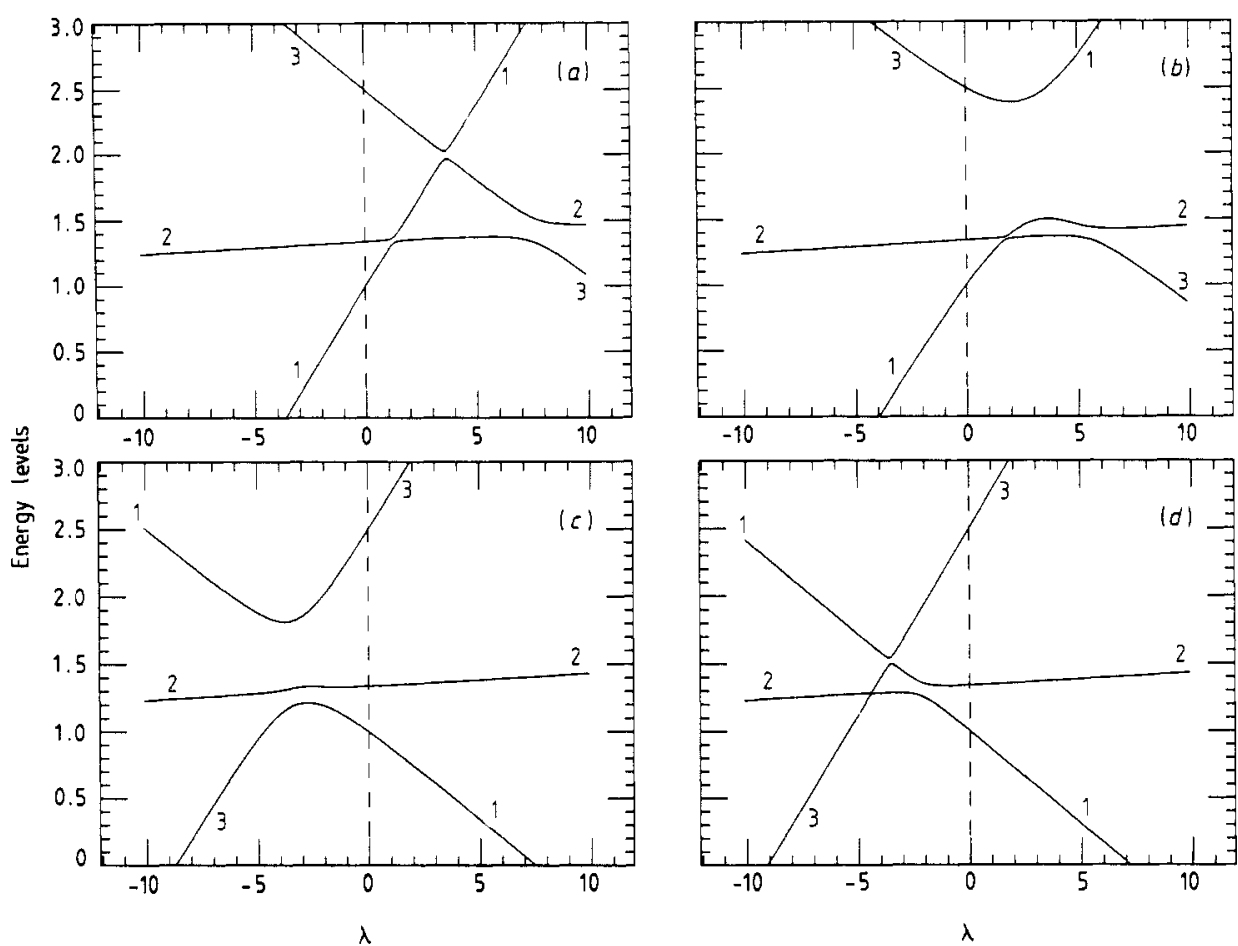

Figure 2. The three levels as a function of $\lambda$ for fixed $\varphi_{1,2}=\varphi_{2,3}=0.08$ while $\varphi_{1,3}$ assumes the values $0.03(a), 0.35(b), 1.35(c)$ and $1.54(d)$. The eigenvalues of $H_{1}$ are $0.275,0.01$ and -0.14 . The significance of the descending order is discussed in section 4 .

The set of angles $\varphi_{1, k}, k=2, \ldots, N$ can be obtained by identifying the first column of $U$ with the column vector

$$
\left(\begin{array}{c}
c_{2} \cdots c_{N} \\
s_{2} c_{3} \cdots c_{N} \\
s_{3} c_{4} \cdots c_{N} \\
\vdots \\
s_{N-1} c_{N} \\
s_{N}
\end{array}\right)
$$

Here we denote by $c_{k}$ and $s_{k}$ the cos and the sin function, respectively, of $\varphi_{1, k}$. The matrix $B_{1}$ has the form

$$
B_{1}=\left(\begin{array}{cccccc}
c_{2} \cdots c_{N} & -s_{2} & -c_{2} s_{3} & -c_{2} c_{3} s_{4} & \cdots & -c_{2} \cdots c_{N-1} s_{N} \\
s_{2} c_{3} \cdots c_{N} & c_{2} & -s_{2} s_{3} & -s_{2} c_{3} s_{4} & \cdots & -s_{2} c_{3} \cdots c_{N-1} s_{N} \\
s_{3} c_{4} \cdots c_{N} & 0 & c_{3} & -s_{3} s_{4} & \cdots & -s_{3} c_{4} \cdots c_{N-1} s_{N} \\
\vdots & \vdots & \ddots & \ddots & \ddots & \vdots \\
s_{N-1} c_{N} & 0 & \cdots & 0 & c_{N-1} & -s_{N-1} s_{N} \\
s_{N} & 0 & \cdots & \cdots & 0 & c_{N}
\end{array}\right)
$$

In the second step the set of angles $\varphi_{2, k}, k=3, \ldots, N$, is determined in a similar way from the second column of $B_{1}^{-1} U$. Note that the latter matrix has only zeros in the 


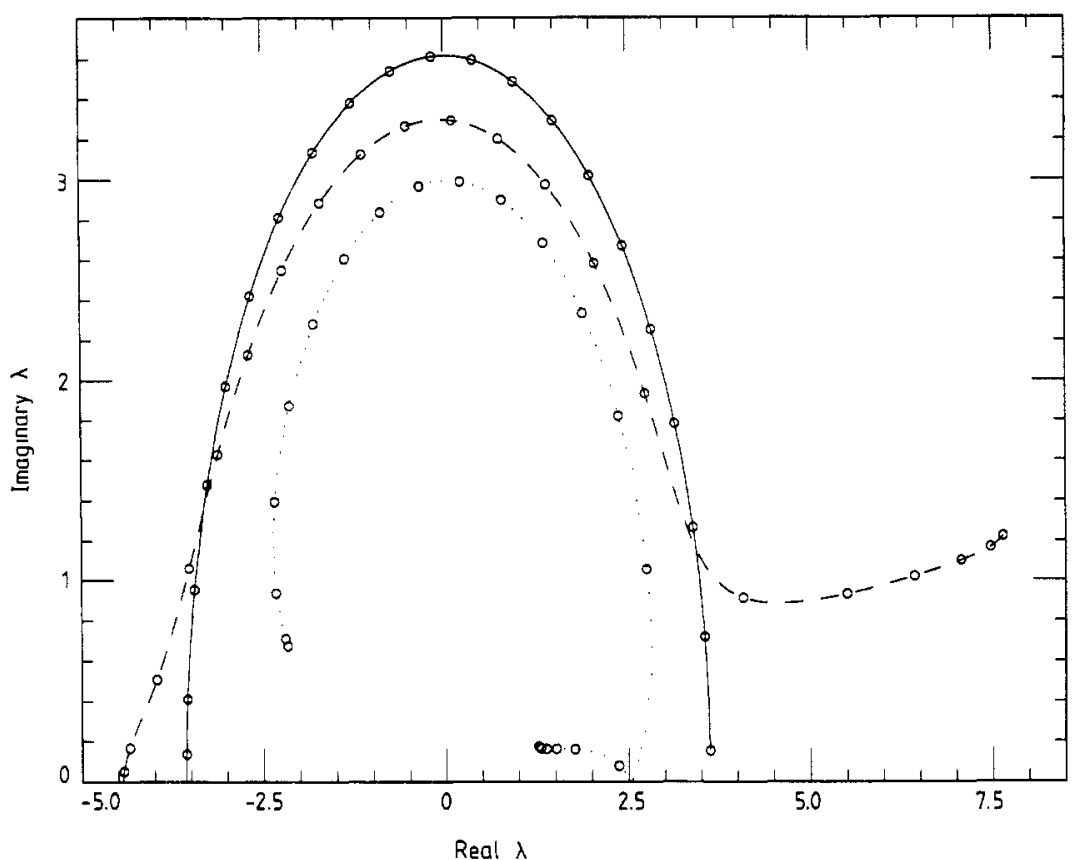

Figure 3. The three trajectories of the exceptional points in the upper $\lambda$ plane obtained from varying $\varphi_{1,3}$ between 0 and $\pi / 2$ with the other two angles kept fixed at 0.08 .

first row and column except for the 1-1 element which is unity. The matrix $B_{2}$ is formed accordingly and $\varphi_{3, k}, k=4, \ldots, N$, is obtained from the third row of $B_{2}^{-1} B_{1}^{-1} U$. This procedure stops when $\varphi_{N-1, N}$ is eventually obtained from $B_{N-2}^{-1} \cdots B_{1}^{-1} U$, yielding $B_{N-1}=R_{N-1, N}$.

In the discussion of the previous section the unperturbed lines are well defined by construction. For a realistic situation the plotting of such lines is not obvious a priori. It appears that the unperturbed lines are well defined only if the matrix $U$ is close to a unit matrix, i.e. when all angles are small. As a first generalisation, we discuss the situation where the angles obtained from $U$ are not small, but they are assumed to be small when calculated from a matrix that is related to $U$ by a suitable permutation and possible sign changes of the columns of $U$. Note that we always refer to $U=U(\infty)$, which means that the order of the column vectors in $U$ relates to an ascending order of the eigenvalues of $H_{1}$.

The proper choice of permutations is best explained by considering the case where $H_{1}$ has very small off-diagonal elements (we recall that we always refer to the basis in which $H_{0}$ is diagonal). This means that the eigenvalues of $H_{1}$ are close to its diagonal elements. If the diagonal elements of $H_{1}$ are all in ascending order, like the order assumed for the $\varepsilon_{k}$, the intersections of the unperturbed lines all occur for negative $\lambda$ values. For positive $\lambda$ values the matrix $U(\lambda)$ is close to a unit matrix, this holds in particular for $U=U(\infty)$. No permutation is needed. In turn, if the diagonal elements of $H_{1}$ are ordered the other way around, all intersections occur for positive $\lambda$ values. For $\lambda$ values larger than the farthest right intersection point, the matrix $U(\lambda)$, and therefore $U$, has a form which is close to

$$
P^{0}=\left({ }_{ \pm 1} \cdot{ }^{ \pm 1}\right)
$$


where the signs depend on the actual order of the intersection points. In this case, many angles turn out to be rather large. However, the matrix $U P^{0}$ is close to a unit matrix and the angles of $U P^{0}$ will be small. This can be generalised. We assume an $H_{1}$ with small off-diagonal elements but no special order of the diagonal elements. This is reflected in the structure of $U$ in that a specific permutation including possible sign changes of column vectors of $U$ yields a matrix $U P$ that is close to a unit matrix. The angles obtained from $U P$ are small, in contrast to those of $U$. The $(k, l)$ element of the matrix $P$ is \pm 1 , if the $k$ th column of $U$ has to be moved into the $l$ th position of $U P$; all other entries of $P$ are zero. The signs of the elements of $P$ are determined by the requirement that $U P$ is close to a unit matrix (this implies $\operatorname{det} P=1$ ). Surely $P$ can be viewed as a rotation as well; however, using the definition of angles as indicated above, there would be considerable arbitrariness in some of the angles; we therefore refer to $P$ as the permutation matrix.

To actually determine $P$ for a given $U$ one searches for the largest (in modulus) element in the $k$ th column. If it occurs in the $l$ th position then the $k$ th column vector of $U$ will be the $l$ th column vector of $U P$. Under the assumption made, this same matrix element will also be the largest in the $l$ th row of $U$. Taking into account the sign of the matrix element, all entries of $P$ are thus determined.

The permutation matrix enables us to draw the appropriate unperturbed lines in a well defined way. For $H_{1}$ we use the form

$$
H_{1}=U P \times P^{-1} D P \times(U P)^{-1} .
$$

The association of the $\varepsilon_{k}$ must now be made with the properly permuted eigenvalues of $H_{1}$ appearing in $P^{-1} D P$. Denoting them by $\omega_{p(k)}$, the unperturbed lines are given by

$$
E_{k}^{0}(\lambda)=\varepsilon_{k}+\lambda \omega_{p(k)}
$$

Since, under the assumption made, all $N(N-1) / 2$ angles of $U P$ are small, a reliable qualitative pattern of the spectrum of $H_{0}+\lambda H_{1}$ can thus be obtained. One has to replace the crossings of the $s$ th with the $t$ th unperturbed line by level repulsions according to the magnitudes of the angles $\varphi_{s, t}$ (obtained from $U P$ ). Depending on the values of the $\varepsilon_{k}$ and the $\omega_{p(k)}$, the level repulsions occur at negative and positive $\lambda$ values. The distribution of the real parts of the exceptional points is essentially given by the intersection points of the $E_{k}^{0}(\lambda)$. The distribution of their imaginary parts is determined by the values of the corresponding $\varphi_{s, t}$.

The diagrams in figure 2 may help to illustrate the point. In section 3 all diagrams have been obtained with a descending order of the elements of the diagonal matrix $P^{-1} D P$ occurring in $H_{1}=U P \times P^{-1} D P \times(U P)^{-1}$. For the top diagrams the angles of $U P$ are small and hence the unperturbed lines are obtained from associating the ascending $\varepsilon_{k}$ with the descending elements of $P^{-1} D P$, where

$$
P=\left(\begin{array}{ccc}
0 & 0 & -1 \\
0 & 1 & 0 \\
1 & 0 & 0
\end{array}\right)
$$

in this particular case. However, for the bottom diagrams the unperturbed lines are obtained from associating the $\varepsilon_{k}$ with the ascending elements of $D$, since the angles of $U$ are now small (note that we have chosen $\varphi_{1,3} \simeq \pi / 2$ for $U P$ ). This is due to the 
fact that the angle $\varphi_{1,3} \simeq \pi / 2$ effectively interchanges $\omega_{1}$ and $\omega_{3}$ as long as the other angles are small.

Basically, the procedure described so far can be applied even if we drop the assumption about the smallness of the off-diagonal elements of $H_{1}$. In the example discussed in the previous paragraph this could correspond to an intermediate value of $\varphi_{1,3}$, i.e. $\varphi_{1,3} \simeq \pi / 4$, or any choice for the other angles. The permutation matrix is defined in principle by the requirement that the angles of $U P$ are minimal. Since an implementation to determine $P$ in this way seems rather involved, we suggest here a heuristic approach which is guided by the procedure indicated above.

In the general case, more than one column vector of $U$ will be found which has its largest component in the same position. Also, the largest, say lth, component of the $k$ th column may not be the largest component of the $l$ th row. And for some values of $l_{0}$ the $l_{0}$ th position may not occur. We propose a procedure of 'second choice' which works efficiently and yields a matrix $U P$ which is sufficiently close to a unit matrix for our purpose. In the first search all columns are allocated their final position, for which the largest component is simultaneously the largest component of the corresponding row. There remain a number of columns of $U P$ which are missing (e.g. the fifth position was never found to be the largest among all columns of $U$ ). This number is equal to the number of columns of $U$ which must find another allocation (e.g. the seventeenth position was found more than once to be the largest). From the latter set of column vectors a reduced square matrix is formed by keeping only the rows with the missing numbers. A second search is carried out with the the reduced matrix. The procedure is iterated. Tests with randomly generated orthogonal matrices for $N=60$ yielded a reduced matrix of typical dimension ten to fifteen in the first step. After three or fewer iterations $U P$ was determined. We mention that we expect more favourable situations in physical cases where both $H_{0}$ and $H_{1}$ are assumed to be regular. In other words, the transformation $U$ which relates the bases of two regular Hamiltonians is expected to be diagonally dominant rather than having random elements.

In section 2 we found $\varphi(-\infty)=\varphi(\infty)+\pi / 2$ and interpreted $U(\lambda)$ as a continuous rotation by the angle $\pi / 2$ when $\lambda$ sweeps over the real numbers. The generalisation to $N$ dimension implies all the $N(N-1) / 2$ angles $\varphi_{i, k}(\lambda)$. A simple statement connecting the angles at $\lambda=-\infty$ with those at $\lambda=\infty$ can no longer be made. The generalisation from two to $N$ dimensions is expressed by the relation

$$
U(-\infty)=U(\infty)\left({ }_{ \pm 1} . .^{ \pm 1}\right)
$$

with a similar matrix as in (6), apart from the signs which depend on the specific sequence of avoided crossings. It is interesting to visualise the behaviour of the rotational matrix $U(\lambda)$ qualitatively as a function of $\lambda$. It describes the motion of an $N$-bein with an orientation given by the two endpoints $U(-\infty)$ and $U(\infty)$, with $U(0)$ being the unit matrix in between. Locally, this motion essentially affects two of the $N$ axes. Globally it appears rather erratic, owing to the $N(N-1) / 2$ level repulsions which give rise to a whirling movement as the individual phases influence each other from their analytic connection via the exceptional points. We mention that in particularly simple models for many-body systems like the Lipkin model [13] $U(\lambda)$ can be a simple monotonic rotation when $\lambda$ is varied [14]; this corresponds to the fact that, while the Lipkin model is of the form $H_{0}+\lambda H_{1}$ and does have $N(N-1)$ exceptional points, it is characterised by a complete lack of fluctuations [15] in the spectrum for all values 
of $\lambda$. Generically, however, this movement is expected to follow an irregular path. A quantitative discussion goes beyond the scope of this paper; a possible approach could rely on the nonlinear coupled evolution equations [12] which connect $E_{k}(\hat{\lambda})$ and the matrix elements of $U(\lambda)$. More insight into the behaviour of the state vectors in connection with level crossing is expected from our viewpoint.

\section{Summary and discussion}

The major result of this paper lies in a statement about the distribution of the exceptional points for the Hamiltonian $H_{0}+\lambda H_{1}$. Their real parts are essentially given by the intersection points of the unperturbed lines. These are obtained when the proper association between the spectrum of $H_{0}$ and the spectrum of $H_{1}$ is established using the permutation matrix $P$ (equation (7)). The values of the angles obtained from $U P$ determine the imaginary parts of the exceptional points.

It appears obvious that a characterisation of quantum chaos hinges on the distribution of the exceptional points. This distribution is primarily governed by the intersection points of the unperturbed lines and secondarily by the values of the angles associated with $U$. Future work will concentrate on first applying our procedure to situations which are acknowledged to be chaotic, such as the hydrogen atom in a strong magnetic field [16]. In this way further insight will be gained about the statistical properties of the distribution of the exceptional points which yields quantum chaos. Based on the experience of such studies, the procedure will be applied to other physical situations where the classical analogy is not amenable.

The aim of this paper is to elucidate the principle. We expect the approach to also be helpful in the understanding of the high sensitivity found in chaotic systems under perturbation. In fact, a perturbation of either $H_{0}$ or $H_{1}$ will in general bring about a redistribution of the exceptional points. Such displacement of the exceptional points may not necessarily change their statistical distribution. However, at least locally, a sensitive behaviour of spectrum and wavefunctions can be expected, owing to the motion of the closely lying singularities. In accordance with the discussion of the erratic behaviour of the matrix elements $U_{m, n}(\lambda)$, we expect a corresponding pattern of matrix elements of, say, the momentum operator under variation of $\lambda$. Likewise, the dependence of $\langle m|p| n\rangle$ on $m$ or $n$ for fixed $\lambda$ is expected to be erratic. Indications to this effect are found in simple models relating to quantum chaos [17].

We are aware that quantum mechanical operators are represented by infinite matrices and that, strictly speaking, quantum chaos can be expected only in the limit $N \rightarrow \infty$. In order to make progress we limited ourseives to the finite-dimensional case, as is the practice in virtually all work related to this subject.

It is not suggested that one determine the precise positions of the exceptional points in a particular case, as this is at least as expensive as a matrix diagonalisation. Future work based upon the ideas presented here must concentrate on statistical statements about (i) the positions of intersection points of straight lines, (ii) the properties of large orthogonal matrices and (iii) the mutual influence of the effect of exceptional points upon the spectrum when they lie close together, but in different Riemann sheets. Up to now there are the two traditional approaches to quantum chaos: the statistical treatment using GOE Hamiltonians and the semiclassical treatment using the classical analogy in small systems. We hope that this paper may provide a bridge between the two. 


\section{Acknowledgments}

One of us (WDH) gratefully acknowledges useful discussions with the organisers and other participants at the workshop on 'Dynamic Properties of Small Quantum Systems' held at Santa Barbara during the first half of 1989 and organised by M C Gutzwiller and E J Heller. This work was supported in part by the National Science Foundation (US) grant no PHY86-04197 and PHY88-17296.

\section{References}

[1] Schukan T H and Weidenmüller H A 1972 Ann. Phys., NY 73108

Davis E D and Heiss W D 1986 J. Phys. G: Nucl. Phys. 12805

[2] Hornbostel K J 1988 Report 333 SLAC

[3] Gering M Z I and Heiss W D 1986 Phys. Rev. D 331980 Quick J and Heiss W D 1987 J. Phys. G: Nucl. Phys. 131201

[4] Casati G (ed) 1985 Chaotic Behaviour in Quantum Systems (New York: Plenum)

[5] Brody T A, Flores J, French J B, Mello P A, Panday A and Wong S S M 1981 Rev. Mod. Phys. 53385

[6] Eckhardt B 1988 Phys. Rep. 163205

[7] Berry M V 1985 Proc. R. Soc. A 400229

[8] Seligman T H and Nishioka H (eds) 1986 Quantum Chaos and Statistical Nuclear Physics (Lecture Notes in Physics 263) (Berlin: Springer)

[9] Gutzwiller M C 1983 Physica 7D 341

[10] Kato T 1966 Perturbation Theory of Linear Operators (Berlin: Springer)

[11] Berry M V 1985 Chaotic Behaviour in Quantum Systems ed G Casati (New York: Plenum)

[12] Pechukas P 1983 Phys. Rev. Lett. 51943 Yukawa T 1985 Phys. Rev. Lett. 541883

[13] Glick A J, Ljpkin H J and Meshkov N 1965 Nucl. Phys. 62199

[14] Heiss W D and Kotzé A A 1988 S. Afr. J. Phys. 1126

[15] Heiss W D 1988 Z. Phys. A 329133 Heiss W D and Kotzé A A 1988 Z. Phys. A 331223

[16] Wintgen D and Friedrich H 1986 Phys. Rev. Lett. 57571 Delande D and Gay J C 1986 Phys. Rev. Lett. 572006

[17] Heiss W D and Kotzé A A 1990 Z.Phys. A in press 